\section{(1) \\ B Cbservatori de \\ Bioètica i Dret}

ISSN: 1886-5887

\section{Revista de Bioética y Derecho}

Publicación del Máster en Bioética y Derecho www.bioeticayderecho.ub.es

ARTíCULO

\title{
Diagnostico genético prenatal y aborto. Dos cuestiones de eugenesia y discriminación
}

\author{
Fabiola Villela Cortés* \\ JORGE E. LINARES SALGADO*
}

Índice

1. Introducción

2. Aborto terapéutico.

3. Aborto con fines sociales. El caso de las niñas en China e India

3.1. El caso de la India

3.2. China. La política del hijo único

4. Aborto eugenésico

5. Selección de sexo y enfermedades ligadas a sexo

5.1. El caso de la hemofilia

5.2. La Distrofia Muscular de Duchenne

6. Crítica al aborto eugenésico. La discriminación de la discapacidad

7. Conclusiones

8. Bibliografía

\footnotetext{
* Dra. Fabiola Villela Cortés. Secretaria académica del posgrado en Filosofía de la Ciencia, UNAM. fabiola.villela@gmail.com

* Dr. Jorge E. Linares Salgado. Coordinador del posgrado en Filosofía de la Ciencia, UNAM. lisjor@unam.mx
} 


\title{
Resumen
}

Los avances en genética seguidos de las nuevas tecnologías en la detección temprana de afecciones genéticas conllevan dilemas bioéticos sobre el uso adecuado de estas técnicas, la información que se le da a la mujer embarazada y la decisión que ella tomará al recibirla. Detectar a tiempo anomalías genéticas permite, en algunas ocasiones, el inicio de un tratamiento adecuado que permita que el niño por nacer no desarrolle una enfermedad discapacitante, como el caso de la fenilcetonuria, o una intervención quirúrgica para un feto con espina bífida, pero en caso que no se tenga ningún tratamiento disponible el aborto sigue siendo la última alternativa. Aunque para muchos cualquier tipo de aborto es eugenésico, esto es busca eliminar individuos no deseados, el objetivo de este artículo es revisar los diferentes tipos de aborto y diferenciar entre el aborto propiamente eugenésico del aborto terapéutico, del aborto con fines sociales o demográficos y del realizado por selección de sexo, para al final proponer que en ciertos casos el aborto eugenésico podría considerarse como bioéticamente aceptable.

Palabras clave: aborto; diagnostico genético prenatal; eugenesia; discriminación.

\begin{abstract}
Advances in genetics followed by the new technologies in the early detection of genetic disorders involving bioethical dilemmas about the proper use of these techniques, the information given to pregnant women and the decision that she will take to receive it. Early detection of genetic abnormalities allow, in some cases, the initiation of appropriate treatment to allow the unborn child does not develop a disabling disease, as the case of phenylketonuria, or surgery for a fetus with spina bifida, but If you do not have any treatment available abortion remains the last alternative. Although for many, any type of abortion is eugenics, that is seeking to eliminate unwanted individuals, the aim of this paper is to review the different types of abortion and the difference between eugenic abortion, therapeutic abortion, abortion for social or demographic ends and Sex-selection made for the for the final proposal that in some cases eugenic abortion could be considered acceptable for bioethics.
\end{abstract}

Key words: abortion; prenatal genetic diagnosis; eugenics; discrimination. 


\section{Introducción}

La carga de trastornos genéticos es importante en todas las partes del mundo [1]. Hay varios factores a considerar en la reproducción humana y la incidencia de trastornos genéticos: la alta tasa de nacimientos, la procreación en una edad reproductiva cada vez mayor, así como la falta de recursos alimenticios y sanitarios básicos que afectan el desarrollo idóneo de un feto, sobre todo en países con grandes problemas de justicia social.

Los adelantos en medicina y particularmente los destinados a la capacidad diagnósticapreventiva son cada vez mayores, esto abre la posibilidad de intervenir cada vez con mayor antelación en el desarrollo de alguna enfermedad o padecimiento. Este hecho genera dilemas éticos en la aplicación de pruebas genéticas y particularmente en aquellas destinadas a detectar enfermedades en fetos, como es el caso del Diagnóstico Genético Prenatal o DGP.

Este diagnóstico ha modificado radicalmente el manejo de los embarazos, ya no es necesario esperar hasta que nazca el bebé para tomar acciones relacionadas con su salud, ahora las afecciones pueden ser detectadas desde el útero. Esto ha provocado nuevos dilemas para el campo de la bioética ente ellos el aborto (legalización o despenalización), las enfermedades que se deben incluir en programas de diagnóstico prenatal, el uso de procedimientos y tratamientos médicos que pueden ir de lo no invasivos (como cambio de dieta materna en caso de fenilcetonuria) a altamente invasivos (como cirugía fetal, fetoscopías, etc.) los cuales que pueden poner en riesgo la vida y salud de la madre y/o del feto, entre otros [2,3].

El Diagnóstico Genético Prenatal o DGP consta de tres etapas: la primera es el asesoramiento genético ${ }^{1}$, la segunda es la aplicación de las técnicas de diagnóstico, como ecografías o fetoscopías ${ }^{2}$ que permiten detectar anomalías anatómicas (como labio leporino, espalda bífida, afecciones cardiacas, etc.) o cromosómicas (como síndrome de Down, trisomía 18, etc.), y la tercera es la toma de decisiones.

Actualmente el diagnóstico genético prenatal es una opción cada vez más accesible y menos invasiva. Generalmente está indicado cuando por lo menos uno de los padres tiene antecedentes de riesgo genético, haber procreado previamente un hijo con enfermedades graves o cuando la edad de

\footnotetext{
${ }^{1}$ El asesoramiento genético es la primera fase del proceso de diagnóstico y se debe considerar la información que se brinde, la tecnología con la que se cuente para poder realizar o no un tratamiento, el acceso a las instituciones que ofrezcan realizar un tamiz genético (por ejemplo en Cuba por derecho el acceso está garantizado y el servicio es gratuito, subsidiado por el Estado), el tipo de enfermedades que se incluyan en un programa de diagnóstico prenatal (deberá ser un problema de salud presente en un porcentaje considerable de la población, del cual se conozca el avance natural de la enfermedad y los posibles tratamientos).

${ }^{2}$ En general las técnicas de diagnóstico genético prenatal se dividen en no invasivas e invasivas. En el primer grupo encontramos Tamizaje bioquímico de cromosopatías (ej. Triple marcador: Alfa Feto Proteína, Gonadotrofina Coriónica Humana, Estriol no conjugado), Tamizaje ecográfico de cromosopatías y defectos estructurales (ej. Ecografía realizada en la semana 10 a 14), Diagnóstico prenatal en células fetales circulantes en sangre matera. En el segundo grupo encontramos Biopsia de vellosidad corial, embriofetoscopía, amniocentesis clásica y precoz, cordocentesis, biopsia fetal, celocentesis, lavado transcervical, entre otros.
} 
la madre supera los 35 años. Idóneamente la finalidad que persigue el DGP es la terapéutica, esto es, tratar la afección detectada.

En la gran mayoría de los casos el diagnóstico prenatal proporcionará tranquilidad a los padres sobre la salud óptima del feto; sin embargo, en los casos restantes la demanda del DGP conlleva a la difícil decisión de proseguir con el embarazo de un feto con taras genéticas o anatómicas, o abortar al feto malformado o con defectos percibidos como difíciles para la vida del niño y del ámbito familiar. Es la toma de decisión la que ha provocado que el Diagnóstico Genético Prenatal levante muchas cuestiones éticas respecto al derecho de decidir quién vive y quién no, y discriminación hacia discapacitados, por mencionar los más difíciles.

Para la mayoría de los médicos católicos, el DGP es considerado una medida eugenésica pues, como habíamos mencionado, en algunas ocasiones conlleva al aborto. Algunos creen que si se prohibiera la interrupción del embarazo, en caso que los resultados del DGP fueran negativos, el interés de las parejas por este método sería menor, lo cierto es que para muchas parejas el DGP seguido del aborto es su mejor opción, pues no todos tienen los medios para mantener un hijo con graves taras en salud. Lamentablemente la opción de tratar in útero al feto no siempre está disponible, ya que no existen tratamientos seguros y/o efectivos para todas las afecciones y peor aún, no en todos los países ni en todos los hospitales se tiene la infraestructura necesaria para ofrecer esta alternativa, ni todos los seguros médicos las cubren, lo que deja a la pareja sin otra opción más que la de abortar.

Uno de los elementos fundamentales en una sociedad democrática, que considere la autonomía de los individuos como esencial, se ve reflejado en el respeto sobre la decisión de una mujer en lo que respecta al embarazo y reproducción. Exigir a una mujer embarazada simpatizante del movimiento pro-vida que aborte es equivalente a exigirle a una mujer a favor del movimiento proelección que no lo haga, ambos son incompatibles con la noción de respeto a la autonomía [4,5].

Este principio (el de respeto a la autonomía) está basado en el respeto a la habilidad de la persona autónoma de controlar su propia vida y a decidir qué sucederá con su cuerpo, con sus planes de vida, con su economía, etc. Queda claro que el aborto es un tema delicado, con muchas aristas y muchas vertientes razón por la cual se ha revisado por expertos en casi todas las áreas de la bioética.

Como ya se señaló, éste es un problema en el que comúnmente se mezclan la discriminación, políticas de salud y fines demográficos. De ahí que sea indispensable hacer dos distinciones: la primera es no confundir aborto terapéutico con el propiamente eugenésico, y en segundo lugar marcar la diferencia ente aborto como medida de control poblacional o con fines sociales. A continuación expondremos las diferencias entre aborto terapéutico, aborto con fines sociales o demográficos ejemplificado con el caso de la India y la política China de un solo hijo, la selección de sexo y finalmente el aborto eugenésico. 


\section{Aborto terapéutico}

Este tipo de aborto se recomienda cuando la continuación del embarazo puede amenazar la vida de la mujer o deteriorar gravemente su salud y el médico no tenga alternativa al no poder salvar a ambos (por ejemplo, si el embarazo es ectópico, esto es, cuando el embrión se implanta en la trompa de Falopio, o si la madre tiene cáncer, hepatitis, cardiopatías, nefropatías, etc.). La evidencia clínica demuestra que el embarazo puede agravar muchos problemas de salud crónicos o eventos traumáticos padecidos por la mujer embarazada; sin embargo, no existe un consenso internacional alrededor de la lista de morbilidades con relación a las cuales la interrupción del embarazo debe ser una opción. Esto ha provocado que el acceso al aborto terapéutico dependa del juicio personal de los médicos, quienes no tienen orientación de los asuntos legales, y de los factores de salud y éticos que deben tomarse en consideración para poder tomar una decisión informada.

Si se considera la salud mental de la mujer, entonces dentro de este apartado de aborto terapéutico se puede incluir aquel que se provoca cuando es resultado de una violación. Esta distinción dependerá de cada país. En el caso de México se ha diferenciado claramente el aborto terapéutico del eugenésico y del provocado por violación.

En México 29 estados declaran en sus códigos penales que este tipo de aborto no es considerado como un delito, salvo los estados de Guanajuato, Guerrero, Chihuahua, Hidalgo y Querétaro. El caso específico del Código Penal de Baja California señala "El aborto no será punible... cuando de no provocarse la embarazada corra peligro de muerte a juicio del médico tratante quien dará aviso de inmediato al ministerio público y este oirá el dictamen de un médico legista, siempre que esto fuera posible y no sea peligrosa la demora" [7].

\section{Aborto con fines sociales. El caso de las niñas en China e India}

Este tipo de aborto está relacionado directamente con los estándares sociales, generalmente la selección de sexo no persigue fines terapéuticos sino económicos. Las parejas prefieren tener hijos varones y algunas están dispuestas a someterse a tratamientos de fertilización asistida para asegurar un niño, no por cuestiones de salud sino por razones económicas, políticas o culturales.

En 1990 el artículo publicado por Amartya Sen titulado "More than 100 million women are missing", refiriéndose a la ausencia de mujeres en el continente asiático ocasionadas por el infanticidio y feticidio femenil, capturó la atención pública y a partir de entonces el tema ha sido estudiado por muchos autores. En este artículo se verán brevemente dos casos, el de la India y China.

\subsection{El caso de la India}

En la India el déficit de mujeres es bastante conocido y las razones de este fenómeno son generalmente económicas. De acuerdo con Beatriz Campos Masilla (2010), durante muchos años se podían ver anuncios publicitarios de clínicas para realizar abortos que señalaban "gaste 500 rupias y 
ahorre 50,000 mañana" lo que quería decir que si se abortaba una niña, se podría ahorrar después una dote [8].

A pesar de que tiene poco que este problema se convirtió en un tema de discriminación mundial, esta práctica se ha venido realizando desde mucho antes. En la segunda mitad del siglo XIX el censo poblacional Indio arrojó estadísticas que indicaban que había más hombres que mujeres. Esta diferencia originalmente se adjudicó a que las mujeres estaban confinadas a las labores del hogar y por tal motivo no habían participado del conteo poblacional, pero poco después se confirmó que las clases medias y altas recurrían, y recurren, al infanticidio y feticidio femenino [9].

Este problema ha crecido tanto que en 1994 se creó una ley que prohíbe informar a las parejas del sexo del futuro bebé mediante. Esta Ley (n57); estipula que ningún centro de orientación genética, laboratorio o clínica practicará técnicas de diagnóstico prenatal con objetivos diferentes a la detección de anomalías cromosómicas, enfermedades genéticas metabólicas, enfermedades genéticas ligadas al sexo y anormalidades congénitas entre otras. Para poder realizar estos estudios la mujer embarazada debe tener más de 35 años, haber sufrido dos o más abortos espontáneos, haber estado expuesta a agentes potencialmente teratógenos ${ }^{3}$ como drogas, radiaciones, infecciones o agentes químicos, contar con un historial familiar de retrasos mentales o deformaciones físicas o genéticas $y$, si después de haber cubierto con lo antes citado, se practica el diagnóstico, bajo ninguna circunstancia se le informará a ella o a sus familiares del sexo del bebé ni verbalmente ni por signos ni de cualquier otra forma [9].

Al parecer, independientemente de la existencia de esta ley, en este país se genera un negocio de 645,000 euros anuales debido a interrupciones voluntarias de embarazo, tanto así que el acrónimo SD que originalmente significa Sex Detection (Detección de sexo) también significa Solution to Dowry (solución a la dote) [8].

Se estima que el infanticidio y feticidio femeninos están provocando un déficit de 48 millones de mujeres en la India y 55 millones en China. Al parecer hay un aumento relativo en las áreas urbanas de India en donde se realizan estas prácticas y donde se registra un mayor desequilibrio poblacional de género es en una zona denominada "el cinturón del feticidio" en los estados de Punjab y Haryana [8].

\subsection{China. La política del hijo único}

El caso de China es probablemente el más sonado, pero, al igual que el caso de la India, esta práctica es antigua y el infanticidio de niñas se tiene documentada desde el 233 a. C. por Han Feizi ${ }^{4}[10]$. Durante décadas se logró manipular la información sobre la falta de niñas en China, al principio se dijo que los padres escondían, literalmente, a sus hijas de los censos poblacionales, pues

\footnotetext{
${ }^{3}$ Del griego teratos que significa monstruo y genos o génesis que significa producción o nacimiento, los teratógenos son sustancias que afectarán al feto produciendo anomalías físicas o genéticas.

4 “Cuando un niño nace, sus padres se felicitan, pero cuando nace una niña, ellos la matan... La razón de esta diferencia en tratamiento es que los padres están pensando en la conveniencia del futuro y calculando lo que a la larga más les convendrá" (Herrlee, 1953, en Benítez, 1986).
} 
estos indicaban que la tasa de nacimientos era normal entre niños y niñas; sin embargo, poco después se hizo evidente que las muertes infantiles en niñas se incrementaban con cada año de vida, reduciendo gradualmente su proporción.

El aborto inducido en este país fue legalizado en 1957 y se convirtió en una práctica común en los 70's a raíz de la campaña de planeación familiar que promovía la disminución de hijos por familia. Para 1979 se implementó la política de "un solo hijo". En la década de 1980, durante la República Popular China, se difundió el uso de ultrasonido lo que provocó que, al conocer el sexo del feto durante el segundo trimestre de embarazo, la práctica de abortos se incrementara, especialmente en las áreas rurales de China. Esta campaña poco después fue modificada, y permitió a las familias de zonas rurales tener dos hijos. Una de las razones para evitar tener una hija, sobre todo en zonas rurales, es que el varón tiene más fuerza física y por tanto puede desempeñar más actividades, más demandantes, lo que se proyecta en cuestiones económicas para la familia. Las condiciones que debe cubrir una pareja para poder tener un segundo hijo son estipuladas en cada provincia, pero casi todas coinciden en que el primer nacimiento haya sido una niña, que el segundo hijo nazca por lo menos cuatro años después del primero, que la mujer tenga más de 28 años de edad y que los dos padres sean, a su vez, hijos únicos [11].

El número de abortos disminuyó considerablemente después de que se implementaron medidas anticonceptivas y mayor información en reproducción sexual; sin embargo, el número de abortos sigue siendo alto y al parecer se suman a los otros mecanismos aún vigentes como el infanticidio, y el cuidado negligente o maltrato a niñas que les provoca la muerte a temprana edad.

A diferencia de la India, el grado educativo en las mujeres chinas no influye en la toma de decisiones, tanto mujeres campesinas con pobre educación como mujeres citadinas con grados universitarios optan por aborto selectivo [11].

En ambos casos queda claro el estatus que tiene el sexo femenino en ambas culturas. La falta actual de mujeres lejos de reubicar el papel social que tienen las hace víctimas de mal trato, cada vez son desposadas más jóvenes, las secuestran de sus casas y en algunas ocasiones son madres a tan corta edad que mueren durante el parto.

Se puede concluir que el diagnóstico genético prenatal seguido del aborto de niñas en estos países no puede ser considerado como prácticas eugenésicas, en ellas se refleja una cuestión determinada por la cultura y factores sociales en estos países (aunque este fenómeno se presenta en varios países europeos y de medio oriente) que afecta considerablemente la salud reproductiva de las mujeres.

\section{Aborto eugenésico}

Una de las principales causales de despenalización y/o legalización del aborto es cuando el producto presenta alteraciones genéticas o congénitas, también denominado aborto eugenésico [12]. En muchas legislaciones existe esta causal para no perseguir, lo que en otras condiciones se consideraría, un delito punible. Actualmente este tipo de aborto no es coercitivo, es decir, quien toma la decisión de llevar a término o no un embarazo en el que el feto presente anomalías genéticas, 
malformaciones o enfermedades graves, es la madre y no el médico, familia o Estado; es una decisión autónoma.

Éste tipo de aborto representa un problema de política de salud pública en países en desarrollo, cuyas legislaciones son, en general, más bien restrictivas y, por tanto, la cantidad de abortos clandestinos, realizados muchas veces en condiciones insalubres, sigue siendo numerosa.

El aborto eugenésico confronta dos posturas opuestas, pro-vida y pro-elección. La primera sostiene que la vida es lo más preciado y que nadie tiene derecho a terminar con ella, en contra parte, la postura pro-elección considera el derecho de decidir de la madre, las posibilidades físicas económicas, sociales o psicológicas, de la madre para poder elegir no tener un niño severamente enfermo.

El problema con este aborto son los límites que han de marcarse para determinar su recomendación o despenalización, entre la vida digna e indigna e inhumana. Generalmente este límite se establece considerando el tipo de taras que presenta el feto, la gravedad de las mismas y si existe un posible tratamiento, ya sea antes o después del nacimiento. La incurabilidad de las enfermedades que provoquen un sufrimiento extremo en el niño por nacer puede ser un límite para recomendar o decidir realizar un aborto eugenésico [13].

\section{Selección de sexo y enfermedades ligadas a sexo}

El objetivo que persigue este tipo de abortos es prevenir el nacimiento de un niño con un importante riesgo de padecer una enfermedad que le provocará gran sufrimiento. Esta situación en particular mezcla la posibilidad de realizar un aborto eugenésico o de optar por un tratamiento. A continuación se presentan dos claros ejemplos de cómo los avances en medicina son cruciales para decidir abortar o no a un feto con enfermedades ligadas al sexo graves, la hemofilia y la Distrofia Muscular de Duchenne.

\subsection{El caso de la hemofilia}

La hemofilia A es un trastorno congénito de la coagulación caracterizado por la deficiencia de factor VIII en la sangre [15]. Esta enfermedad ocurre en todos los grupos raciales, con una incidencia aproximada de 1:10 000. La hemofilia severa se caracteriza por tener un nivel de coagulación menor a $1 \%$ de los normales, quienes la padecen presentan hemorragias espontáneas y recurrentes en articulaciones (rodillas, codos, tobillos). Si no se trata a tiempo estas hemorragias pueden generar artritis discapacitante a temprana edad. Asimismo, presentan hemorragias en músculos y tejidos blandos [15].

Los genes del factor de coagulación VIII y IX (involucrados en la presencia de hemofilia) se encuentran en el cromosoma $X$, de ahí que esta enfermedad se encuentre ligada al sexo, por tanto casi todos los pacientes son varones (salvo que una niña herede ambos cromosomas $\mathrm{X}$ recesivos, en este caso ella presentará la enfermedad). Las hijas de varones con hemofilia son portadoras obligadas y tienen un $50 \%$ de probabilidad de transmitir el padecimiento a un hijo y $50 \%$ de tener una 
hija portadora. Una tercera parte de los casos surgen en familias que no tienen historia previa de la enfermedad, lo que indica nuevas mutaciones [15].

Antiguamente los pacientes morían antes de la etapa reproductiva debido a las hemorragias y sus complicaciones. La medicina, en su esfuerzo por darles una mejor calidad de vida, provocó que se incrementara el periodo de vida, permitiendo que algunos pacientes llegaran a la edad reproductiva y tuvieran descendientes. Actualmente los pacientes varones con hemofilia, si bien es cierto que su estado de salud es delicado, pueden integrarse a la escuela, conseguir un trabajo y, en general, tener una buena calidad de vida. Por tal motivo, la mujer embarazada puede decidir continuar con el embarazo de un varón hemofílico, o bien decidir únicamente tener hijas.

Una de las ventajas que ofrece la terapia génica de células somáticas ${ }^{5}$ a estos pacientes es que podrán tener hijos varones que presentarán la enfermedad pero esta podría ser tratada a tiempo, permitiéndole llevar una vida normal. Aunque este panorama sigue estando en el ámbito de la especulación, lo cierto es que los tratamientos para los pacientes hemofílicos cada vez son mayores, lo que permite que no todas las mujeres, al conocer que su hijo presenta esta enfermedad, decidan abortar.

\subsection{La Distrofia Muscular de Duchenne}

Esta distrofia es la más grave y recuente de las distrofias musculares progresivas que afecta a uno de cada 3500 varones nacidos [16]. Se caracteriza por una degeneración lenta de las fibras musculares esqueléticas, relacionada con la deficiencia de la proteína distrofia, que lleva al paciente a la invalidez dentro de los primeros 10 años de vida y provoca la muerte por inanición, insuficiencia respiratoria ${ }^{6}$ y/o cardiaca ${ }^{7}$ en la segunda década de la vida [17], los pacientes pueden presentar un promedio cognitivo inferior al normal que se manifiesta más claramente en déficit del lenguaje [16].

Esta enfermedad, al igual que la hemofilia se encuentra ligada al sexo, el gen responsable se encuentra en la región 21 del brazo corto del cromosoma X, este cromosoma presenta altas tasas de mutación conocida, por lo que la tercera parte de los individuos afectados no presenta antecedentes familiares. Puesto que no existen aún tratamientos terapéuticos eficientes para esta enfermedad, la estrategia más utilizada es el aborto eugenésico.

\footnotetext{
${ }^{5}$ Consiste en el tratamiento, prevención y eliminación de alguna enfermedad mediante la transferencia de ADN o el uso de genes para reemplazar genes alterados. Su objetivo es corregir el origen genético de una enfermedad y no sólo los síntomas. Los primeros protocolos fueron planeados para corregir enfermedades con un origen genético hereditario bien definido, mediante la inserción funcional del gen que evitara la expresión del gen defectuoso. Actualmente el uso de esta tecnología se ha extendido a enfermedades con diferente origen ya sea infeccioso, alérgico, auto-inmune y el tratamiento del cáncer. En especial la terapia génica en células somáticas se aplica a las células capaces de mantener las funciones corporales normales. No afecta a las células reproductivas (óvulo, esperma u óvulo fecundado) y por tanto no pasa a la siguiente generación. Las personas con tratamiento en células somáticas aún pueden transmitir el desorden o enfermedad a sus descendientes.

${ }^{6}$ Hipoventilación crónica alveolar en estado avanzado

${ }^{7}$ Cardiomiopatía dilatada y/o arritmias cardiacas
} 
Un estudio realizado en Brasil que analizó la percepción de la calidad de los pacientes con DMD reportó que la causa principal de infelicidad es el aislamiento social que tienen los pacientes, resultado de la inmovilidad propia de la enfermedad. Actualmente, los avances en nuevos tratamientos incluyen, terapia con células madre, trasplante de mioblastos, terapia genética de remplazo, terapia sobre-reguladora, inhibidores de proteosoma, entre otras. Lamentablemente estas alternativas se encuentran en etapa experimental y los resultados son diversos. Hasta el momento solo se tienen tratamientos quirúrgicos, de rehabilitación y algunos medicamentos que no han probado su eficiencia. Mientras no exista un tratamiento curativo sólo se puede retrasar la evolución de la enfermedad [16].

Hasta ahora las opciones reproductivas que tienen las mujeres portadoras son: la adopción, tratamiento de Fertilización in vitro (FIV) con donante de ovocitos y posterior diagnóstico genético preimplantacional (DGPI), arriesgarse a tener un hijo que presente la enfermedad y, en caso que el diagnóstico genético prenatal (DGP) revele que el feto varón presenta la mutación, optar por un aborto eugenésico, o simplemente no tener hijos.

\section{Crítica al aborto eugenésico. La discriminación de la discapacidad}

La discusión moral sobre el aborto es bastante problemática en muchos países, pues hasta el momento no existe un consenso social amplio sobre cuándo puede aplicarse, cuándo penalizarse y cuándo no, ni si debe prevalecer el derecho a la vida sobre el derecho de la mujer de decidir sobre su propio cuerpo o viceversa. El caso del aborto eugenésico no es la excepción, pues por un lado se considera el bienestar del feto y, por otro, diversas comunidades de minusválidos lo perciben como una discriminación o negación del valor que la sociedad da a vidas y cuerpos diferentes al de la mayoría. Debemos recordar que en todos los movimientos eugenésicos gubernamentales del siglo XX uno de los objetivos claros era detener el nacimiento de personas que pudieran traer alguna discapacidad física o mental, pues se les consideraba como un "lastre social". Actualmente es el término "calidad de vida" lo que está en juego. En este punto, Bone [13] se pregunta "¿Habrá una creciente intolerancia hacia una menor calidad de vida?", esto es, ¿una intolerancia a la minusvalía?

El uso de técnicas como el DGP representa para la comunidad discapacitada un eslabón más en la cadena de abusos dirigidos a sus personas. La retórica de progreso genético encierra discriminación social y económica al menospreciar la vida de los discapacitados catalogándola de pasiva, trágica o no digna [18]. De acuerdo con Buchanan [18], el concepto de progreso que reside en el corazón mismo de la ideología de la nueva genética discrimina y devalúa a los individuos discapacitados, y los afecta con la mayor injusticia posible: rechazar el derecho a su existencia.

No debemos olvidar que muchas veces la decisión final que puedan tomar los padres o la madre de un feto con afecciones graves va a estar influida, tanto por la información que le ofrezca el ginecobstetra como por presiones y prejuicios sociales. Darrin Dixon (2008), en su artículo titulado Informed consent or institutionalized eugenics?, analiza la situación del aborto en fetos diagnosticados con Síndrome de Down en EUA, y muestra que la balanza se inclina hacia el rechazo de abortos, mencionando varios factores que influyen en las decisiones de las parejas o mujeres para no abortar, uno de ellos es el acceso a atención médica. Señala que en Pittsburg, EUA, se realizan 
menos abortos por esta causa que en el resto de EUA, debido a que en esta ciudad hay un mayor porcentaje de católicos, hay más servicios para atender a los niños con discapacidades y la ley federal protege a todos los niños discapacitados. Los padres reciben servicios gratuitos hasta que el niño cumple tres años, edad en la que comienza un plan educacional individual que le permite desarrollar ciertas habilidades para posteriormente asistir a la escuela. Como resultado de estas políticas públicas, los niños con Síndrome de Down tienen mejores oportunidades de vivir, volverse independientes de sus padres y convertirse en parte integral de la comunidad [19]. Esto nos indica que, en efecto, hay ciertas discapacidades que no impiden al individuo que las padece tener una calidad de vida adecuada.

Con este ejemplo vemos claramente que en muchos casos la discapacidad es una cuestión de discriminación, pues no se considera la calidad de vida que puede tener una persona, sino las taras e impedimentos sociales que ésta tendría en una sociedad determinada. En cualquier caso, dependerá de los padres o la madre considerar si cuentan con las condiciones adecuadas para criar a un hijo discapacitado o no, pues no se compara tener un niño con síndrome de Down en Pittsburg que en la Ciudad de México (a pesar del Teletón y esfuerzos de organización no gubernamentales de asistencia privada).

Considero que la discusión en torno al rechazo y discriminación que sufren los discapacitados es válida, siempre y cuando se trate de enfermedades que permitan a la persona que las padece tener una calidad de vida aceptable, pero no tiene cabida cuando un bebé padece alguna enfermedad que, lejos de brindar una calidad de vida adecuada, le provocará sufrimiento durante toda su vida.

Una posible solución a este problema es, por un lado, establecer las diferencias entre enfermedad y discapacidad pues la primera no necesariamente está implicada en la segunda [19] y, por otro, cuestionar la ilusión de que los discapacitados son de facto incompetentes. Pero este tema es tan vasto que se requeriría de mucho más tiempo para poder abordarlo y lamentablemente rebasa los límites de esta investigación.

\section{Conclusiones}

Nuestra propuesta es que un límite para determinar el aborto eugenésico sea hasta dónde la madre o los padres están dispuestos a enfrentar una enfermedad con su hijo. Si para ella o ellos la vida es lo más preciado y por tanto, cualquier enfermedad que presente ese hijo no es un motivo para considerarla como "una vida indigna de ser vivida" sino por el contrario merece la pena vivirla a pesar del sufrimiento que esta pueda provocarle no sólo al hijo(a) sino a ellos mismos, entonces no hay ninguna razón que justifique un aborto eugenésico. La expresión más grande de la autonomía es decidir tener un hijo enfermo. Sin embargo, si la situación es opuesta y la madre o los padres consideran que una enfermedad es incompatible con la noción de calidad de vida o vida digna de ser vivida o no podrán proporcionarle los tratamientos necesarios para paliar el sufrimiento vivido, entonces su decisión de abortar deberá ser respetada.

Esto último lo sustentamos basándonos en el estudio de las demandas realizadas sobre todo en EUA conocidas como "demandas por vida injusta" (wrongful life) en la que los padres demandan a 
médicos y profesionales de la salud en nombre de su hijo(a) por infringir el daño moral de haberlo traído al mundo. Generalmente se argumenta que los padres no recibieron la información necesaria respecto a la enfermedad del hijo(a) antes, durante o después del nacimiento de éste y que la negligencia del personal de salud ocasionó que los padres concibieran o no recurrieran al aborto eugenésico. Por tanto, según ellos, el daño infringido al menor consiste en el hecho de haberlo traído al mundo bajo las condiciones de salud precaria y alto sufrimiento en el que vive actualmente [14]. El 6 de diciembre de 2010 la corte de Bruselas determinó que los padres pueden realizar estas demandas en Bélgica ${ }^{8}$.

\section{Bibliografía}

1. Wertz, D.C., Fletcher, J.C. Berg, K. (2003). Review of ethical issues in Medical Genetics, Human Genetics Program, Management of noncommunicable diseases, World Health Organization, 110p.

2. Casagrandi, D. et al, (2005). Algunos aspectos éticos del diagnóstico prenatal, la medicina y terapia fetales. Revista Cubana de Obstetricia y Ginecología. 31(3)

3. Cabrera, C. (2008). Aspectos bioéticos del diagnostico prenatal. Revista de Obstetricia y Ginecología de Venezuela. 68(3):141-143.

4. Bennett, R. (2008). Is reproductions women's business? How should we regulate regarding stored embryos, posthumous pregnancy, ectogenesis and male pregnancy? Studies in Ethics, Law and Technology. 2(3):1-19.

5. Brock, DW. (2002). Obligaciones éticas para prevenir daños transmitidos genéticamente En Hansberg, O., Platts, M. (comps) (2002) Responsabilidad y libertad, FCE-IIF-UNAM, 49-67p.

6. Artículo 136 del Código Penal del Estado de Baja California Sur, II libro, 1a sección, Título $1^{\circ}$

7. GIRE. Grupo de Información en Reproducción Elegida. www.gire.org.mx

8. Campos, B. (2010). El feticidio e infanticidio femeninos. Nómada. Revista Crítica de ciencias Sociales y Jurídicas 27(3) Disponible en http://www.ucm.es/info/nomadas/27/beatrizcampos.pdf

9. Dobernig, M. (2003) La selección de sexo: una alternativa de las técnicas de reproducción asistida. Jurídica. Anuario del Departamento de Derecho de la Universidad Iberoamericana, 33:

10. Herrlee G.C. (1953), Chinese Thought from Confucius to Mao Tse-Tung, citado en Benítez, 1986, Infanticidio femenino en China, Estudios de Asia y África, El Colegio de México, XXI:3

11. Potts, M. (2006). China's one child policy, BMJ, 333:361-2.

12. González de León, D., Billings, D., 2002, El aborto en México. Ipas. 1-24.

13. Reviriego, F. (1998). Otro estudio más sobre el aborto. La indicación eugenésica y su fundamentación. Cuadernos Bartolomé de las Casas (8): 111

\footnotetext{
8 Para mayor información léase la nota http://www.lifesitenews.com/news/belgium-court-allows-parents-to-sue-forwrongful-birth-when-disabled-child/
} 
14. Brock, DW. (2002). Obligaciones éticas para prevenir daños transmitidos genéticamente en Hansberg, O., Platts, M. (comps). (2002). Responsabilidad y libertad, FCE-IIF-UNAM, 49-67p.

15. Giangrande, P. (2003). Embarazos en mujeres con trastornos de la coagulación hereditarios. Tratamiento de la hemofilia, Federación Mundial de la Hemofilia. 29:1-15.

16. Cammarata-Scalisi, F., Camacho, N., Alvarado, J., Lacruz-Rangel, MA. (2002). Distrofia muscular de Duchenne, presentación clínica. Rev Chil Pediatr. 79(5): 495-501.

17. Rodríguez, M., Ferreira, R., Gayol, L.A., Quintana, J., Rendón, R., Méndez, D. (1996). Diagnóstico de distrofia muscular de Duchenne mediante análisis del ácido desoxinucleotico y su aplicación en la prevención. Rev Cubana Pediatr 68(1): 10-20. Disponible en: http://scielo.sld.cu/scielo.php?script=sci arttext\&pid=S0034-75311996000100003\&lng=es

18. Mittra, J. (2007). Marginalising 'eugenic anxiety' through a rhetoric of 'liberal choice': a critique of the House of Commons Select Committee Report on reproductive technologies. New Genetics and Society. 26(2):159-179.

19. Darrin P. D. (2008). "Informed Consent or Institutionalized Eugenics? How the Medical Profession Encourages Abortion of Fetuses with Down Syndrome" ExpressO

Available at: http://works.bepress.com/darrin dixon/1 